\title{
Cerebrospinal Fluid and Blood Cultures in a Paediatric Population
}

Rosalind Saunders ${ }^{1}$, Simon Hewson ${ }^{1}$, Srini Bandi²

${ }^{1}$ Department of Clinical Microbiology, ${ }^{2}$ Leicester Children's Hospital; University Hospitals of Leicester NHS Trust, Leicester, UK

\section{Background}

Leicester Children's Hospital serves a population of approximately 200,000 children. The aim of this retrospective survey was to evaluate the activity of the Hospital in terms of microbiological investigation of suspected invasive bacterial infections, in order to understand local epidemiology and inform future service development and training needs.

\section{Methods}

The microbiology laboratory results database was searched for all cerebrospinal fluid (CSF) samples and blood cultures taken from paediatric patients $($ age $<16)$ over a 1-year period (1 March 2016 - 28 February 2017)

Samples referred for testing from other Trusts and post-mortem samples were excluded, as were any discarded samples (e.g. leaking, unlabelled). Data recorded included demographic data, requesting location and microbiology result.

All CSF samples receive urgent manual microscopy. Samples with an elevated white cell count (or elevated white:red cell ratio) have a white cell differential count. All CSF samples have a Gram stain and culture performed.

Blood culture samples are cultured on an automated system; bottles that flag positive receive Gram staining and manual microscopy, followed by culture.

\section{Results}

Numbers and collection locations of samples are shown in the table below:

\begin{tabular}{||l|l|l|}
\hline Sending location & CSF & Blood \\
\hline General paediatrics & $352(45 \%)$ & $2139(38 \%)$ \\
\hline Neonatal units & $336(43 \%)$ & $1766(31 \%)$ \\
\hline Paediatric intensive care & $35(5 \%)$ & $480(8 \%)$ \\
\hline Emergency Dept / other & $59(7 \%)$ & $1317(23 \%)$ \\
\hline Total samples & $\mathbf{7 7 7 ( 1 0 0 \% )}$ & $\mathbf{5 7 0 2 ( 1 0 0 \% )}$ \\
\hline No. of unique patients & 738 & 3879 \\
\hline
\end{tabular}

\section{Results - CSF}

40 of 777 CSF samples had positive microscopy (5.1\%). 9 samples $(1.1 \%)$ had bacterial organisms seen on Gram stain $(n=1)$ or cultured $(n=8)$. Three of these were deemed significant (see table), with 6 others of doubtful significance when correlated clinically (skin and oral microbiota).

\section{Significant CSF-positive cases}

Enterobacter sp. in CSF and blood culture in neonate treated for line sepsis and meningitis.

Gram-negative bacilli seen in CSF with E. coli in blood culture in neonate treated for $\mathrm{E}$. coli meningitis.

Streptococcus infantarius in CSF and blood cultures of child in intensive care treated for neonatal sepsis and meningitis.

Nine CSF samples were sent away to a reference laboratory for meningococcal and pneumococcal PCR testing, with one positive result for Neisseria meningitidis serogroup $B$

This study did not record virology results for this set of samples.

\section{Results - Blood}

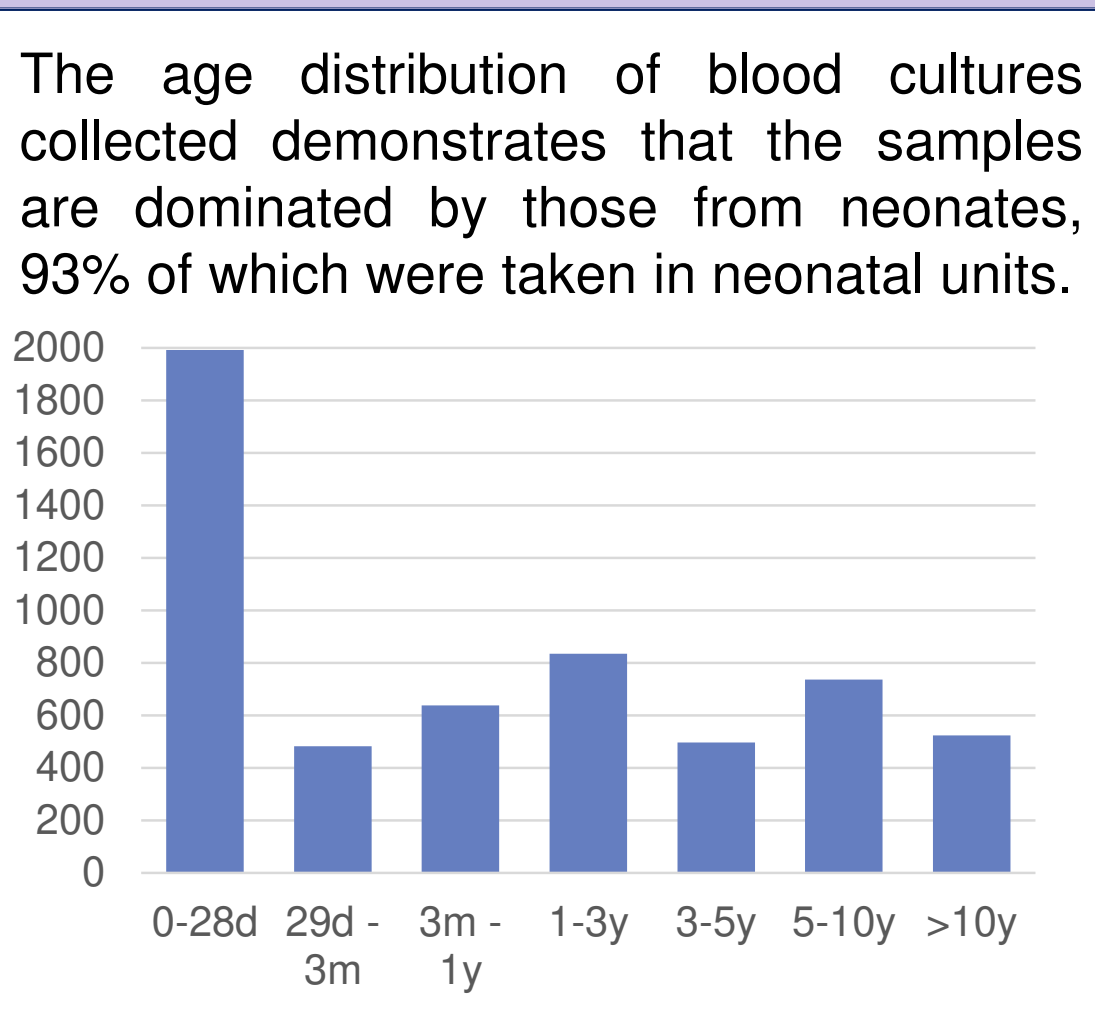

Overall, 316 samples were positive on culture $(5.5 \%) .220$ of these were potential contaminants (including 185 coagulasenegative staphylococci, as well as various skin and oral bacteria of doubtful significance once clinically correlated). The 96 remaining samples had pathogenic organisms:

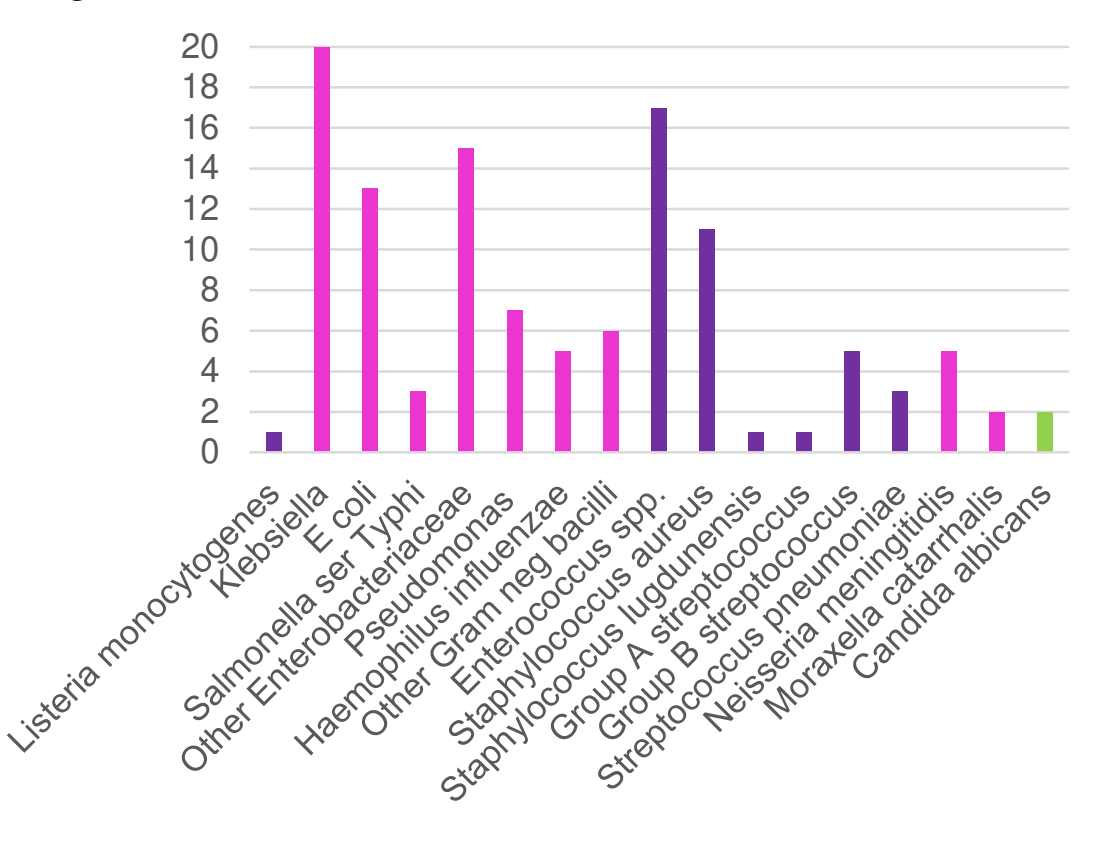

Two complex patients with recurrent bacteraemia contributed to 20 positive samples (Klebsiella, Enterobacter).
PCR testing of blood revealed a further 2 cases of $N$. meningitidis and 5 cases of $S$. pneumoniae.

Antimicrobial resistance in this population was not problematic:

All Staphylococus aureus isolates were sensitive to flucloxacillin.

All Streptococcus pneumoniae isolates were sensitive to penicillin.

All Salmonella serovar Typhi isolates were sensitive to ceftriaxone.

All Enterobacteriaceae and Pseudomonas $\mathrm{sp}$. were sensitive to meropenem.

\section{Conclusions}

The yield of significant culture-positive results from sterile sites in children is very low, with the majority of organisms isolated from paediatric CSF and blood being skin/oral commensals and thus potential contaminants.

Nevertheless, the information obtained from significant positive cultures remains clinically valuable. PCR-based methods appear successful in identifying further cases, but pose logistical and financial challenges, and do not yield information on antimicrobial sensitivity to guide treatment.

Continuing education aimed at reducing rates of sample contamination is ongoing in our Trust, in order to ensure that time and resources are appropriately targeted. 